\title{
Beam Fragmentation in Heavy Ion Collisions with Realistically Correlated Nuclear Configurations
}

\author{
M. Alvioli*, M. Strikman \\ The Pennsylvania State University, 104 Davey Lab, University Park, Pennsylvania 16803, USA
}

(Dated: August 16, 2018)

\begin{abstract}
We develop a new approach to production of the spectator nucleons in the ultra-relativistic heavy ion collisions. The energy transfer to the spectator system is calculated using the Monte Carlo based on the updated version of our generator of configurations in colliding nuclei which includes a realistic account of short-range correlations in nuclei. The transferred energy distributions are calculated within the framework of the Glauber multiple scattering theory, taking into account all individual inelastic and elastic collisions using an independent realistic calculation of the potential energy contribution of each of the nucleon-nucleon pairs to the total potential. We show that the dominant mechanism of the energy transfer is tearing apart pairs of nucleons with the major contribution coming from the short-range correlations. We calculate the momentum distribution of the directed flow of emitted nucleons which is strongly affected by short range correlations including its dependence on the azimuthal angle. In particular, we predict a strong angular asymmetry along the direction of the impact parameter $\boldsymbol{b}$, providing a unique opportunity to determine the direction of $\boldsymbol{b}$. Also, we predict a strong dependence of the shape of the nucleon momentum distribution on the centrality of the nucleus-nucleus collision.
\end{abstract}

PACS numbers: 25.75.-q,25.75.Dw,24.10.-i,21.60.Ka

\section{INTRODUCTION}

In this paper we start a program of studies of the nuclear fragmentation in ultra-relativistic heavy ion collisions using as a starting point an event generator of the nucleon configurations in nuclei which correctly reproduces short-range correlations between the nucleons.

Most of the recent experimental and theoretical studies of the relativistic heavy ion collisions were focused on the production of hadrons at central rapidities. Fragmentation of nuclei in these collisions was used only as a supplementary trigger for centrality. At the same time experiments at Relativistic Heavy Ion Collider (RHIC) have demonstrated that it is possible to determine on the event by event basis impact parameter and reaction plane of the collision. (There is obviously some inherit uncertainty related to the fluctuations of the observables for collisions at a given impact parameter.) This opens new opportunities for studies of the nuclear fragmentation which have a long history; see, for example, Ref. [1] and references therein.

Another motivation is the recent direct observation of the short-range correlations (SRC) 2 [5] in the nuclear decays initiated by a hard removal of the nucleon from the nucleus. When combined with the scaling of the ratios in $x>1\left(e, e^{\prime}\right)$ nuclear reactions, it demonstrates the important role played by SRC in nuclear structure; for a recent review, see Ref. [6]. This calls for a description of heavy ion collisions using realistic configurations of nucleons in nuclei going beyond the commonly used collection

\footnotetext{
*Present address: $\mathrm{ECT}^{*}$, Strada delle Tabarelle 286, I-38123, Vil-
} lazzano (TN) Italy of nucleons randomly distributed in the nuclear volume.

We started the program of including SRC in nuclear configurations in Ref. [7] where we implemented central correlation functions using the Metropolis method. This allowed us to overcome the problem of distortion of single particle density when configurations with nucleons at short distance are simply discarded. The pair distribution function for the generated configurations is close enough to the realistic one; in particular, it vanishes when the two nucleons separation approaches zero.

In Ref. [7] the inclusion of correlations in nuclear configurations was shown to have significant effects on the fluctuations of the average number collisions in $N A$ scattering; the authors of Ref. [8, 9] confirmed the importance of $N N$ correlations effects on fluctuations in $N A$ as well as $A A$ collisions using our central-correlated configurations [10] within their Monte Carlo (MC) simulations. In this work we implement the second step of our program: taking into account spin and isospin dependence in the generation of configurations. This allows us to implement for the first time, the state-dependent correlations; this procedure is discussed in Sec. III

In this paper we apply the MC method for taking into correlation effects in AA collisions at the energies of the CERN NA49 experiment 11], also using results from the Lawrence Berkeley National Laboratory (LBL) experiment of Ref. [12]. We analyze dependence of nuclear fragmentation on centrality of the collisions. In the data analyzes the centrality is usually determined based on the the correlation between the observed charged particle multiplicity and the calculated number of participant nucleons in the $A A$ collision. This leads to large uncertainties and the impact parameter is usually known in large bins and very central collisions are difficult to identify. We propose in this work that one can obtain 

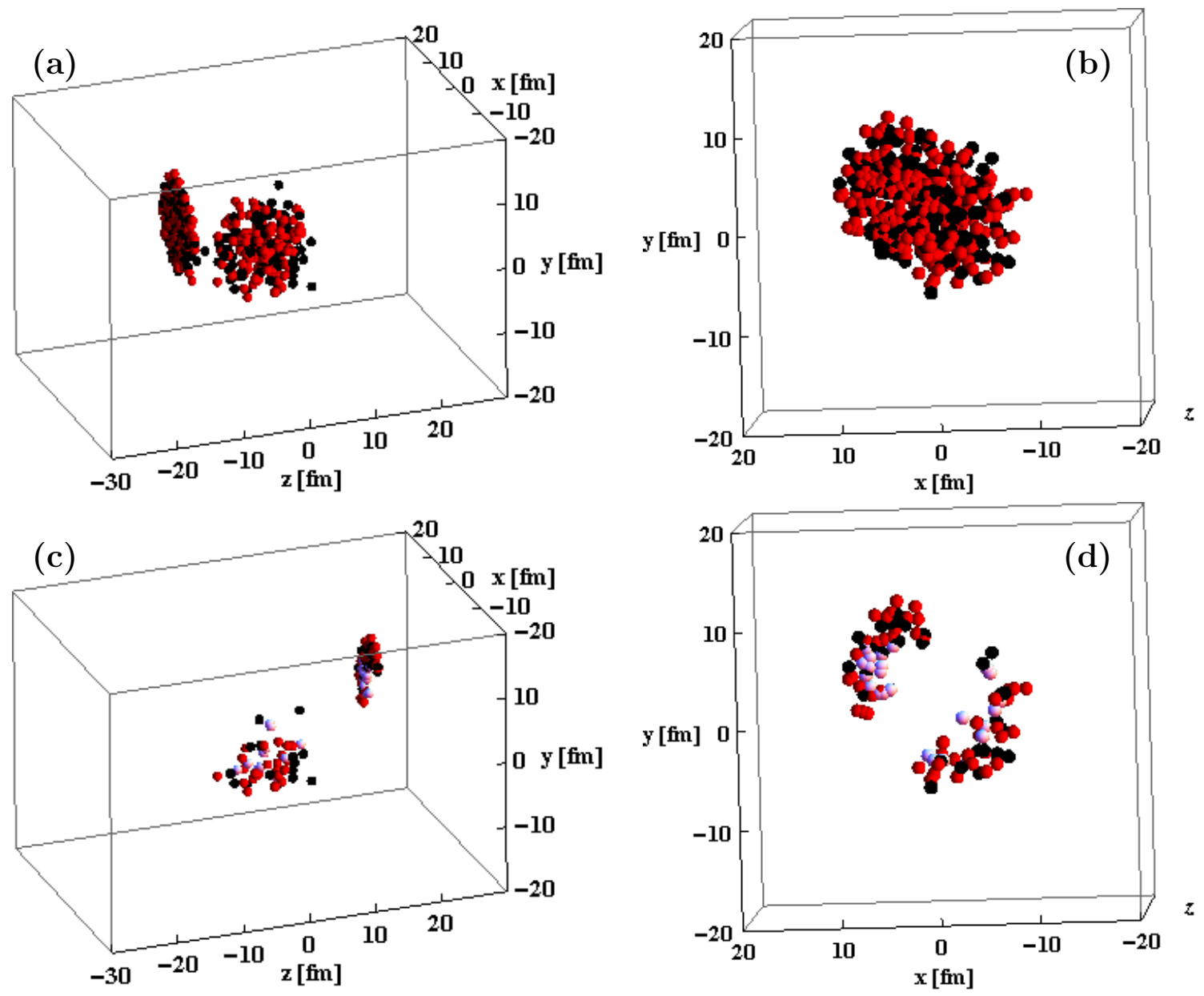

FIG. 1: (Color online) Sketch of a $\mathrm{Pb}-\mathrm{Pb}$ collision in the target rest frame at impact parameter $b=5 \mathrm{fm}$ oriented at $45^{\circ}$ with respect to the $x$ and $y$ axes; the projectile moves along the $z$ axis. [(a) and (c)] View along the beam line; [(b) and (d)] view from behind; [(a) and (b)] before interaction; [(c) and (d)] after interaction. The inelastically interacting nucleons have been removed from the figure. Black and red spheres represent protons and neutron, respectively, while the white ones are active nucleons (see text). The dimension of the spheres are taken as the rms charge radius of the proton. Animations are available at the URL in Ref. [10] along with the configurations used for the colliding nuclei.

additional information about the centrality of a collision, based on the detailed balance of energy transferred in the collision at a given impact parameter and on the emission of high-momentum nucleons originating from SRC pair in nuclei. In addition, the angular asymmetry for emission of nucleons allows us to resolve the sign ambiguity of the direction of impact parameter $\boldsymbol{b}$ in contrast with the current procedures which determine only $|\boldsymbol{b}|$.

We introduce a new model for the description of the emission mechanism of spectator nucleons in ultra-highenergy heavy ion collisions. In this limit, in the rest frame of one of the nuclei, the projectile is strongly Lorentz contracted to the longitudinal size of the order $\leq 1 / \mu$ where $\mu$ is a soft strong interaction scale $\leq m_{\rho}$. As a result, the collision can be considered as the propagation of a "pancake" moving with the speed of light which consequently removes nucleons from the target along its path; the situation is depicted in Fig. 1. It is worth emphasizing that the dynamics of nuclear fragmentation at low energies differens significantly; energy transfers to individual nucleons are small as compared to the scale of energies in SRC, and the relative velocities of nuclei are small. This differs markedly from the picture of a thin pancaked nucleus going through the target nucleus we can employ in the ultra-relativistic collisions. For a review of models of fragmentation at low energies, see Ref. 13]. The spectator system emerging from the target is left in an unknown excited state. At the first stage the destruction of the potentials between the pairs (triplets) of nearby nucleons, one of which is hit and another belongs to the spectator system, release energy on the layer of nucleons adjacent to the removed portion of the nucleus. This energy comes from the work performed by destruction of the potential energy (bonds) associated with the position of these nucleons in the initial wave function, and it can be readily converted into kinetic energy. As a result, they can be emitted into free space in the direction of the removed nucleons, or propagate into the 

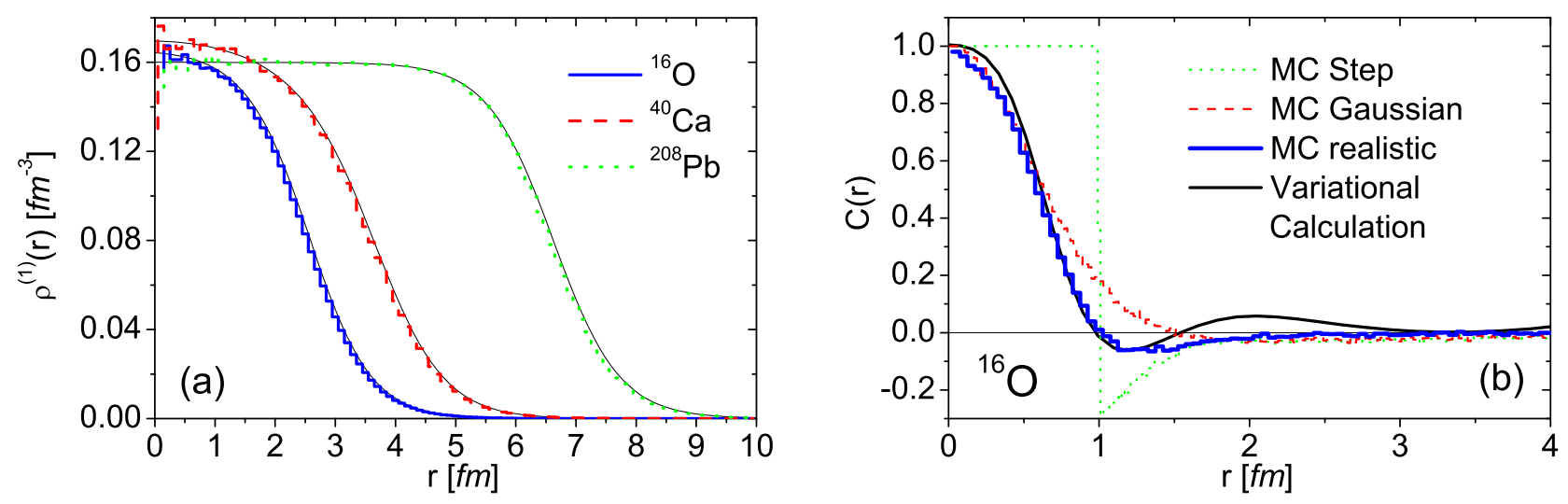

FIG. 2: (Color online) (a) The one-body density of ${ }^{16} \mathrm{O},{ }^{40} \mathrm{Ca}$ and ${ }^{208} \mathrm{~Pb}$; the colored curves correspond to the calculation with our fully correlated configurations, while the black curves are the corresponding input functions. (b) The pair distribution function $C(r)$ of ${ }^{16} \mathrm{O}$ defined in Eq. (2), obtained with $\mathrm{MC}$ within various approximations for the nucleon-nucleon correlation functions contained in Eq. (1), compared with the variational calculation of Ref. [17]. Calculations for ${ }^{40} \mathrm{Ca}$ and ${ }^{208} \mathrm{~Pb}$ nuclei produce similar results.

spectator system and undergo attenuation. The remaining available potential energy goes into excitation of the spectator system and it can be released at a later stage by standard nucleon evaporation and decay. Experimental analyses along the lines we suggest in this work are feasible at RHIC as presence of the directed flow $v_{1}$ for neutrons was observed (though not analyzed in detail) by the PHENIX [14] and STAR [15, 16] experiments.

The paper is organized as follows. In Sec. II we introduce general definitions and describe our improved procedure for generating nucleon configurations in nuclei, which, in difference from our original procedure, includes spin and isospin nucleon-nucleon correlations. We also describe the MC procedure for the first stage of the collision which separates nucleons into spectators and interacting nucleons. It employs the geometry of the Glauber description of the collision, which allows an impact-parameter-dependent description and takes into account the basic features of realistic calculations of SRCs in the ground state of nuclei [17]. (Note here that the Glauber model forms a basis of many MC codes for the simulation of high energy nucleus-nucleus collisions; for example, HIJING [18] for the simulation of nucleus-nucleus collisions.) In our model we use the standard description of hadron-nucleus interactions [19], in which inelastic collisions of nuclei are treated as an incoherent superposition of the individual collisions of the nucleons of the two systems. The participants are defined as the nucleons which interacted inelastically at least once in the $A A$ collision, and spectators are the nucleons which did not interact. They are determined within the Glauber approximation and the simulation of the collisions is performed starting from random nucleons distributed according to a given probability density for the nucleus profile.
In Sec. III we perform first dynamical calculation of the total excitation energy of the spectator system based on the total potential energy due to the links between spectators and interacting nucleons and use of the ana$\log$ of the Koltun sum rule. Inclusion of SRC is critical at this point. Indeed, the realistic calculations of the fractions of total potential energy due to the different $p p$ and pn pairs in nuclei based on the method of Ref. [17] show that, while in the mean field approximation the two contributions are proportional to the corresponding number of pairs, the inclusion of correlation drastically changes these fractions, bringing the $p n$ pairs to carry about $83 \%$ of the total potential energy. The results are given as a function of the impact parameter of the $A A$ collision.

In Sec. IV we first describe the algorithm for the selection of correlated nucleons among the nucleons close to the collision surface. Next, we outline the procedure used to generate emission of both high momentum and low-momentum nucleons from the surface. The soft part of the distribution is due to heating and evaporation of the residual system which includes taking into account extra heating from absorption of the part of the nucleons emitted from the surface by the bulk of the spectator system. We describe also an additional contribution to the nucleon production due to elastic scattering of nucleons near the surface of the collision which is enhanced for the emission angle close to $70 \div 90^{\circ}$ in the nucleus rest frame. The section concludes with numerical results for the nucleon momentum distributions. The relative roles of different mechanisms discussed in the paper is presented as well as and their dependence on the impact parameter.

In Sec. $\mathrm{V}$ we discuss a novel feature of the proposed mechanism of nucleus fragmentation: the strong angular dependence of the nucleon emission. It is due to a large 

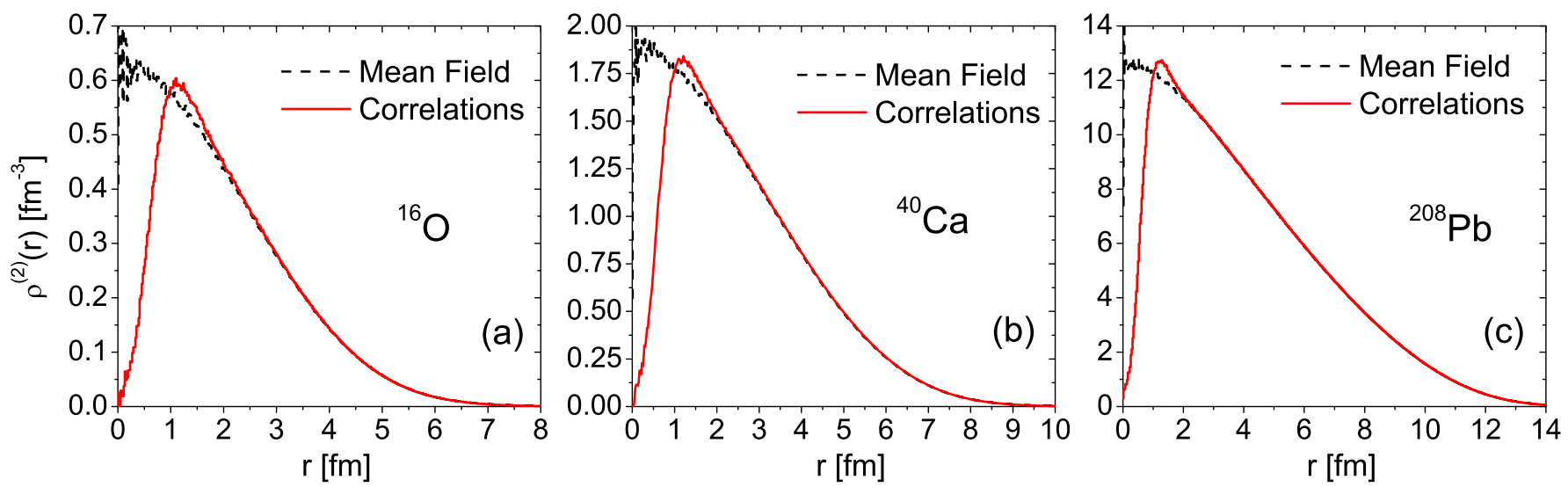

FIG. 3: (Color online) The two-body density matrix defined in Eq. (9) calculated for $(a){ }^{16} \mathrm{O},(b){ }^{40} \mathrm{Ca}$, and $(c){ }^{208} \mathrm{~Pb}$ within our $\mathrm{MC}$ code. The comparison of the uncorrelated results (dashes) and the full correlation results (full lines) for the first operator $O_{12}^{(n=1)}$ is shown in the various panels. The information on state-dependent correlations is contained in the configurations used in the calculation, which were produced using the correlation functions of Ref. [17]. The curves are normalized to $4 \pi \int r^{2} d r \rho^{(2)}(r)=A(A-1) / 2$.

contribution of the emission from the inner surface generated by removal of a fraction of nucleons due to the collision, which is strongly dependent on the geometry of the process. We expect an azimuthal asymmetry which can be exploited to determine the centrality of a given collision event and also resolve the sign ambiguity of the impact parameter vector.

In Sec. VI we compare our results with those of the previous models. Of particular interest is the analysis of Ref. [1], in which the abrasion-ablation model is used to define the participant-spectator mechanism and the subsequent spectator system decay and to perform the estimate of the excitation energy per spectator nucleon. We find that our results for the average characteristics of the nucleon emission are close to the result of Ref. [1] where certain inputs from the data were used.

\section{GENERAL DEFINITIONS AND METHOD}

The inclusion of central correlations in nuclear configurations can be achieved within a Monte Carlo Metropolis method by using as a probability function the square of the wave function of the system taken in the following form:

$$
\psi_{0}\left(\boldsymbol{x}_{1}, \ldots, \boldsymbol{x}_{A}\right)=\hat{F}\left(\boldsymbol{x}_{1}, \ldots, \boldsymbol{x}_{A}\right) \phi_{0}\left(\boldsymbol{x}_{1}, \ldots, \boldsymbol{x}_{A}\right),
$$

where $\phi_{0}\left(\boldsymbol{x}_{1}, \ldots, \boldsymbol{x}_{A}\right)$ is a Slater determinant of singleparticle densities, the vector $\boldsymbol{x}_{i}=\left\{\boldsymbol{r}_{i} ; \sigma_{i} ; \tau_{i}\right\}$ contains the spatial, spin, and isospin degrees of freedom, and the correlation operator $\hat{F}$ can, in principle, include the dependence on all the state-dependent operators contained in realistic nucleon-nucleon potentials; in Ref. 7] a simple product of central correlation functions was considered,
$\hat{F} \equiv F=\prod_{i<j}^{A} f\left(r_{i j}\right)$. The calculation which includes the full product $\hat{F}=\prod_{i<j}^{A} \hat{f}_{i j}$ with $\hat{f}_{i j}$ state-dependent correlation functions, is a formidable task; instead, one can consider an expansion of that product whose first term contains correlation links of the particle under investigation in the Metropolis search with a second particle only, disregarding correlation links between the second particle and the others. The subsequent terms which include such third- and higher-order correlations, namely three-body clusters linked by spin and isospin dependent two-body correlations, as well as genuine three-body correlations, will be neglected in the present work. At the same time the central correlations will be retained to all orders. This procedure is dictated by the enormous computing power needed for a higher-order calculation and justified by the fact that realistic calculations based on the cluster expansion technique show that higher-order calculations, essential for the accurate determination of quantities such as binding energy and momentum distributions, provides corrections to the bulk properties of the quantities of relevance, namely diagonal one- and radial two-body densities, which are small as compared to the accuracy needed by our study. Instead, we will make use of realistic results obtained within the cluster expansion method ([17, 20]) when applicable. The one-body densities obtained with our configurations for $A=16,40$ and 208 are shown in Fig. 2(a). They coincide with very high precision with the analytic function used as an input. In Fig. 2(b) we present the pair distribution function

$$
C(r)=1-\rho_{C}^{(2)}(r) / \rho_{U}^{(2)}(r),
$$

with $\rho_{C}^{(2)}$ and $\rho_{U}^{(2)}$ being the correlated and uncorrelated two-body radial densities, respectively. The procedure of Ref. [7] with improved configurations described above 


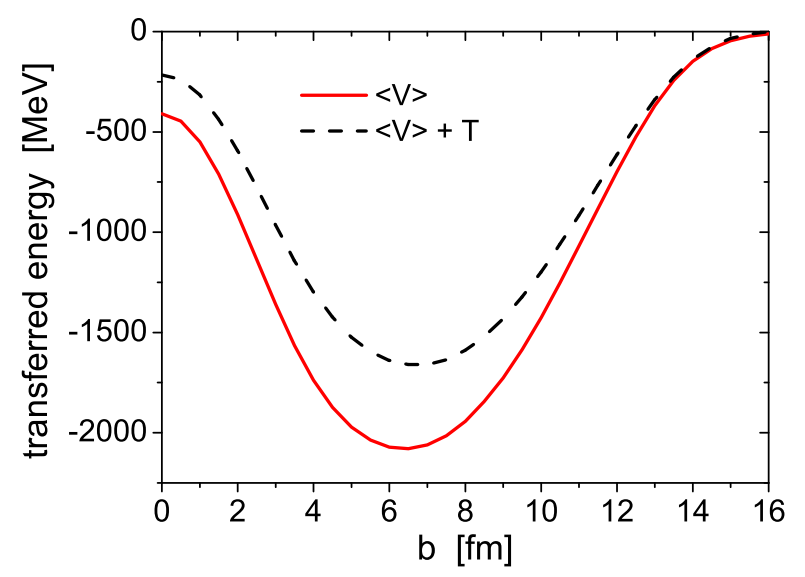

FIG. 4: (Color online) Transferred energy in $P b-P b$ collisions at $P_{L a b}=160 \mathrm{GeV}$, calculated within our model at different impact parameters. (Solid line) Potential energy due to removing of nucleons with no other effects taken into account; (dashed line) transferred energy after (i) subtraction of kinetic energy of emitted high-momentum nucleons and soft nucleons from the interaction surface and (ii) extra energy gained from nucleons absorbed the spectator system.

leads to a better agreement with the realistic variational calculation from Ref. [17] than the original procedure which included only central correlations. For comparison we also the results of Ref. 7] obtained in MC with step ( $\theta$ function) and Gaussian nucleon-nucleon correlation functions which strongly deviate from the realistic calculation already for $r \sim 1 \mathrm{fm}$.

It is possible to describe the bulk features of the geometry of the the nucleus-nucleus collisions using the Glauber multiple-scattering model. Within this framework, nucleons are frozen in their positions during the interaction, which is supposed to be instantaneous. For a given impact parameter of the colliding nuclei, the impact parameter $\boldsymbol{b}_{i j}=\boldsymbol{b}_{i}-\boldsymbol{b}_{j}$ of the $i$-th projectile and $j$-th target nucleons are considered, and their inelastic interaction is evaluated on the event-by-event basis using the impact parameter representation for $N N$ collisions which leads to the probability of the inelastic nucleon - nucleon at a relative impact parameter $b$ :

$$
P_{\text {in }}(b)=1-\left(1-\Gamma\left(\boldsymbol{b}_{i}-\boldsymbol{b}_{j}\right)\right)^{2},
$$

where $\Gamma\left(b_{i j}\right)=\sigma_{N N}^{t o t} \exp \left(-b_{i j}^{2} / 2 B\right) / 4 \pi B$ is the usual nucleon-nucleon elastic profile function. In most of our numerical studies reported below we used the parameters $\sigma_{N N}^{t o t}=39 \mathrm{mb}$ and $B=13.59 \mathrm{GeV}^{2}$, corresponding to NA49 energy. The main effect we neglect here is inelastic shadowing corrections which arise in the Gribov-Glauber approximation - these effects primarily affect interactions far from the interaction surface. We also give results for the energies of RHIC and the Large Hadron Collider (LHC). For illustration purposes, we show in Fig. 1 the

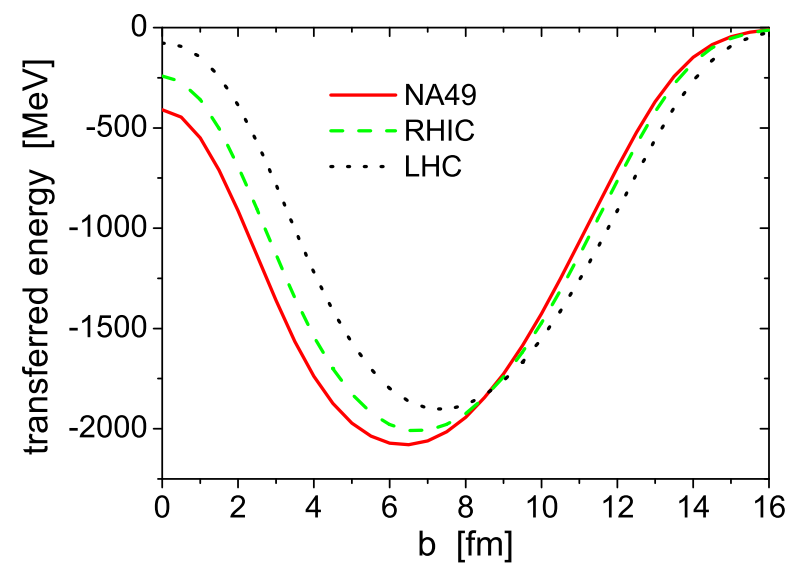

FIG. 5: (Color online) Transferred energy in $\mathrm{Pb}-\mathrm{Pb}$ collisions due to the only potential energy transfer. The values of the Glauber parameters correspond to NA49 (Solid line), RHIC (dashed line) and LHC (dotted line) energies.

spectator nucleons after a $\mathrm{Pb}-\mathrm{Pb}$ collision, for two particular projectile-target configurations. The figure shows, in addition to protons and neutrons (respectively black and red online), nucleons which before the event were correlated with an interacting nucleon. These are shown explicitly in white. The correlated pairs were identified by checking their relative distance and choosing correlated nucleons on the basis of the potential energy they can gain due to removal of the neighboring nucleons, as described in the next sections.

\section{POTENTIAL ENERGY CALCULATION}

The first aim of our analysis is to evaluate the energy transferred to the spectator system in the collision of two heavy nuclei (this calculation does not depend on the details of the fragmentation discussed in the following sections and could be used in any other models of the high energy nuclear fragmentation). To this end, we considered the method of the cluster expansion of Refs. 17] and [20] and found that (i) the inclusion of correlations in the calculation of the potential energy brings the fraction of the total potential energy $\langle V\rangle_{N N}$ due to $p n$ pairs to about $85 \%$ (with the main contribution due to the existence of tensor interactions in the nucleon-nucleon potential) and (ii) the state-dependent radial two-body densities $\rho_{n}^{(2)}(r)$ obtained in Refs. [17] and [20] can be incorporated into our Monte Carlo code to calculate the total energy transferred to the spectator system in a particular collision and to determine the fraction of the energy transfer due to the SRCs.

The potential energy contribution to the ground state 


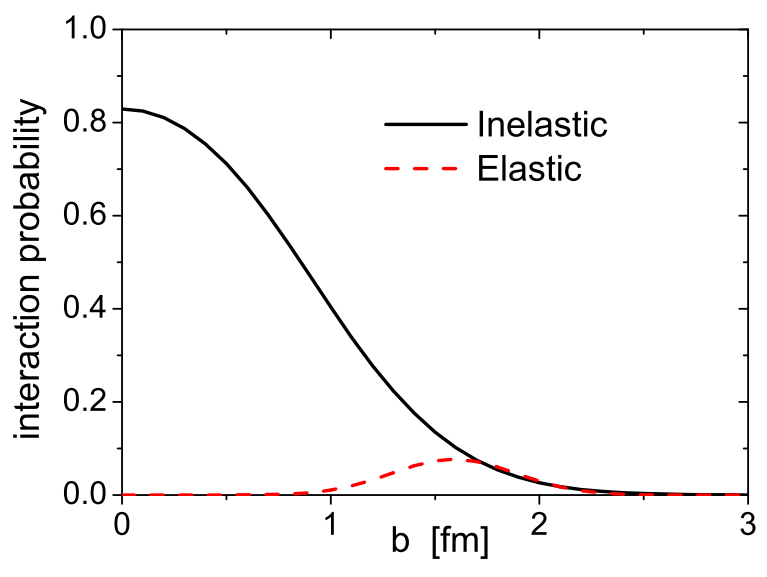

FIG. 6: (Color online) Comparison between the probability of inelastic interaction $P_{i n}(b)$ (solid line) of Eq. (3) and the elastic one $P_{e l}(b)$ (dashed line), shown in Eq. (12), for values of the Glauber parameters corresponding to the experiment of Ref. [11].

energy can be calculated according to

$$
\langle V\rangle=\frac{A(A-1)}{2} \sum_{n=1}^{6} \int d \boldsymbol{R} d \boldsymbol{r} \rho_{n}^{(2)}(\boldsymbol{R}, \boldsymbol{r}) v^{(n)}(r),
$$

where $\boldsymbol{R}=\left(\boldsymbol{r}_{1}+\boldsymbol{r}_{2}\right) / 2, \boldsymbol{r}=\boldsymbol{r}_{1}-\boldsymbol{r}_{2}$, and $\rho_{n}^{(2)}\left(\boldsymbol{r}_{1}, \boldsymbol{r}_{2}\right)$ is the state-dependent two-body density matrix defined as

$\rho_{n}^{(2)}\left(\boldsymbol{r}_{1}, \boldsymbol{r}_{2}\right)=\int \sum \prod_{j=3}^{A} d \boldsymbol{r}_{j} \psi^{\star}\left(\boldsymbol{x}_{1}, \ldots, \boldsymbol{x}_{A}\right) \hat{O}_{12}^{(n)} \psi\left(\boldsymbol{x}_{1}, \ldots, \boldsymbol{x}_{A}\right)$

Here the sum extends over all the discrete degrees of freedom so the final result is a spin-isospin-averaged quantity. Equation (5) was evaluated within the cluster expansion method [17]. $\hat{O}_{12}^{(n)}$ are the operators

$$
\hat{O}_{12}^{(n)} \in\left\{\hat{1}, \boldsymbol{\sigma}_{1} \cdot \boldsymbol{\sigma}_{2}, \hat{S}_{12}\right\} \otimes\left\{\hat{1}, \boldsymbol{\tau}_{1} \cdot \boldsymbol{\tau}_{2}\right\}
$$

acting between particles 1 and 2, which inlcude spin- and isospin-dependent nucleon-nucleon potential and corresponding operators in the nucleus ground-state wave function. Hence Eq. (11) can be rewritten as

$$
\begin{array}{r}
\psi_{0}\left(\boldsymbol{x}_{1}, \ldots, \boldsymbol{x}_{A}\right)=\prod_{i<j}^{A} \hat{f}_{i j} \phi_{0}\left(\boldsymbol{x}_{1}, \ldots, \boldsymbol{x}_{A}\right)= \\
=\prod_{i<j}^{A} \sum_{n=1}^{6} f^{(n)}\left(r_{i j}\right) \hat{O}_{i j}^{(n)} \phi_{0}\left(\boldsymbol{x}_{1}, \ldots, \boldsymbol{x}_{A}\right) .
\end{array}
$$

The two-body density appearing in Eq. (4) can be easily separated, within the cluster expansion as well as in the
$\mathrm{MC}$ calculation, into the contributions due to protonproton $(p p)$, proton-neutron $(p n)$ and neutron-neutron $(n n)$ pairs:

$$
\begin{aligned}
& \rho_{n}^{(2)}\left(\boldsymbol{r}_{1}, \boldsymbol{r}_{2}\right)= \\
& =\rho_{p p}^{(2, n)}\left(\boldsymbol{r}_{1}, \boldsymbol{r}_{2}\right)+\rho_{p n}^{(2, n)}\left(\boldsymbol{r}_{1}, \boldsymbol{r}_{2}\right)+\rho_{n n}^{(2, n)}\left(\boldsymbol{r}_{1}, \boldsymbol{r}_{2}\right) .
\end{aligned}
$$

The potential energy can thus be written as a sum of three contributions corresponding to $p p, n n$, and $p n$ contributions ( $p p$ and $n n$ contributions are identical since the same single particle orbitals have been used both for protons and neutrons states). Calculations show that for central correlations (the total result in this case has a wrong absolute value due to the lack of realistic correlations needed to approximate the experimental values of binding energies), the individual contributions for $p p$ and $p n$ pairs are exactly proportional to the fraction of $p p$ pairs, (23\%), and pn pairs, (53\%), respectively. At the same time, if the full correlations are included, this proportionality no longer holds: the $p p$ contribution represents $8 \%$ of the total, and the $p n$ the $83 \%$ of the total; if we translate this to the contributions of isospin 0 and 1 pairs, we obtain a ratio of $74 \%$ for $I=0$ and $26 \%$ for $I=1$. These features of the short-range nuclear structure can be exploited within the formalism we are going to describe.

We now outline how to incorporate the information presented in this section in our MC code. The realistic calculation of Ref. [17] provides us with the contribution to the total potential energy of a given pair of nucleons, while the corresponding calculation of total kinetic energy from momentum distributions for medium-weight nuclei, in Ref. [17], and for heavy nuclei, in Ref. [21], can be used to evaluate the total excitation energy of the nucleons which do not experience inelastic interactions.

For each $A A$ event, we select spectator and interacting nucleons in both nuclei, using Eq. (3) as the interaction probability; this quantity depends on the (nucleon pair) relative impact parameter $b_{i j}$. Accordingly the probability not to interact is given by

$$
P_{i}^{\text {survival }}\left(b_{i}\right)=\prod_{j=1}^{A}\left(1-P\left(b_{i}-b_{j}\right)\right)
$$

Hence we can calculate for a given event the potential energy which is freed by instantaneous removal of the inelastically interacting nucleons. It represents the amount of the energy available for freeing nucleons from the bound state and generating their kinetic energy (in a sense our approximation resembles the Koltun sum rule 22] for removal of one nucleon from the nucleus). The total potential energy of the nucleus can be calculated in terms of the two-body density of the system or, more specifically, from the radial two body-density defined as follows:

$$
\rho_{n}^{(2)}(|\boldsymbol{r}|)=\int d \boldsymbol{R} \rho_{n}^{(2)}\left(\boldsymbol{r}_{1}=\boldsymbol{R}+\frac{\boldsymbol{r}}{2}, \boldsymbol{r}_{2}=\boldsymbol{R}-\frac{\boldsymbol{r}}{2}\right) .
$$



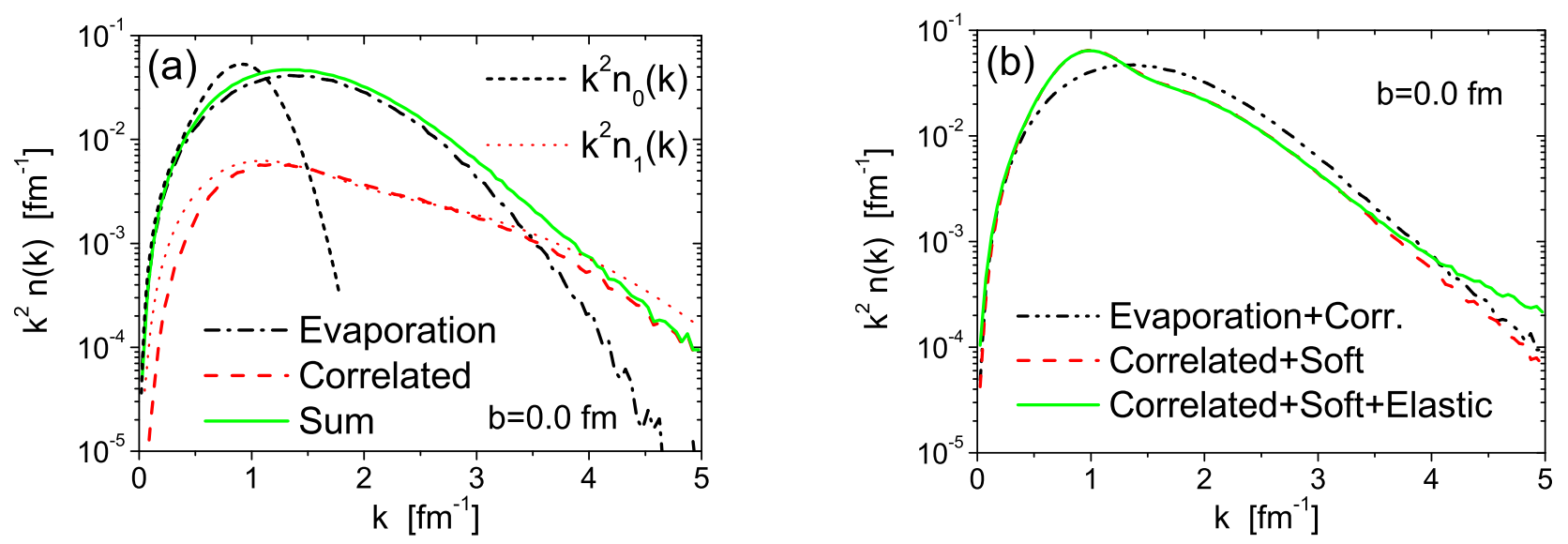

FIG. 7: (Color online) The momentum distributions $k^{2} n(k)$ of directed flow of emitted nucleons in $P b-P b$ collisions at $P_{L a b}=160 \mathrm{GeV}$, calculated within our model at $b=0$ within different approximations. (a) Comparison of the input momentum distributions $n_{0}(k)$ and $n_{1}(k)$ against the MC output considering only evaporation and emission of correlated nucleons (no primary elastic scattering and no additional soft nucleons emitted from the surface taken into account); the contribution of the high-momentum tail of $n_{1}(k)$ is evident in the sum. (b) The total momentum distributions from MC within the various approximations described in the text. The normalization of each curve is set to the actual number $N$ of nucleons falling into the corresponding definition, according to $4 \pi \int d k k^{2} n(k)=N$.

This quantity may be easily calculated in the MC approach by considering all the pairs and building the distribution of their relative distances. It is then straightforward to calculate the fractions of the total two-body potential energy which are due to the interaction between two spectators, two nucleons which experienced inelastic collisions, and mixed pairs. Comparing these fractions with the realistic calculations, in (small) intervals in $r$ corresponding to the bins used in the Monte Carlo determination of the densities, properly scaling the $\mathrm{MC}$ densities to the ones obtained in the realistic calculations and rescaling to the experimental value of the binding energy of the initial nucleus, we can define the fractions $\left\langle V>_{N N}^{S P E},\left\langle V>_{N N}^{I N T}\right.\right.$ and $\left\langle V>_{N N}^{M I X}\right.$ accordingly. The MC procedure is not accurate enough as far as the state-dependent radial two body densities $\rho_{n}^{(2)}$ are concerned for a meaningful determination of the expectation value of the realistic, state dependent potential $\hat{V}_{i j}=\sum_{n} v^{(n)}\left(r_{i j}\right) \hat{O}_{i j}^{(n)}$ (such a calculation would require a much more accurate balance of several positive and negative parts which is currently practical) The procedure can also be applied to the individual $p n$ and $p p$ pairs. The two-body densities obtained from the generated nuclear configurations are presented in Fig. 3 for different nuclei. The figure shows both the results corresponding to uncorrelated and correlated configurations for the radial two-body density associated with the central operator, namely $\rho_{n=1}^{(2)}$ in Eqs. (5) and (10).

The outlined procedure determines the fraction of potential energy due to mixed pairs of nucleons on the event-by-event basis. The results of the calculation of this quantity (which determines the total excitation energy of the spectator system which is released in the process of nucleon / fragment emission) are presented in Fig. 4 for the case of collisions at the momentum of $160 \mathrm{GeV} / c$ per nucleon for which the most extensive high energy studies of the fragmentation were performed. We plot the potential energy due to the disrupted pairs and the same (negative) quantity plus the (positive) kinetic energy due to the emission of high-momentum nucleons. Fig. 4 also shows the dependence of the transferred energy as a function of the impact parameter. Also, we show in Fig. 5 the dependence of the transferred energy on the value of the cross section of $N N$ interactions taken for values corresponding to NA49, RHIC and LHC energies. One can see that for average impact parameters the energy dependence is rather weak. At the same time, for small impact parameters, the energy transfer drops with an increase of energy since the chances for spectators to survive decrease due to increase of $\sigma_{N N}^{t o t}$, while the increase of $\sigma_{N N}^{t o t}$ leads to increase of the probability to interact with several nucleons at large impact parameters, leading to the increase of the energy transfer at large $b \geq 10 \mathrm{fm}$.

The details of our model for the description of emission of nucleons are given in the next sections. 


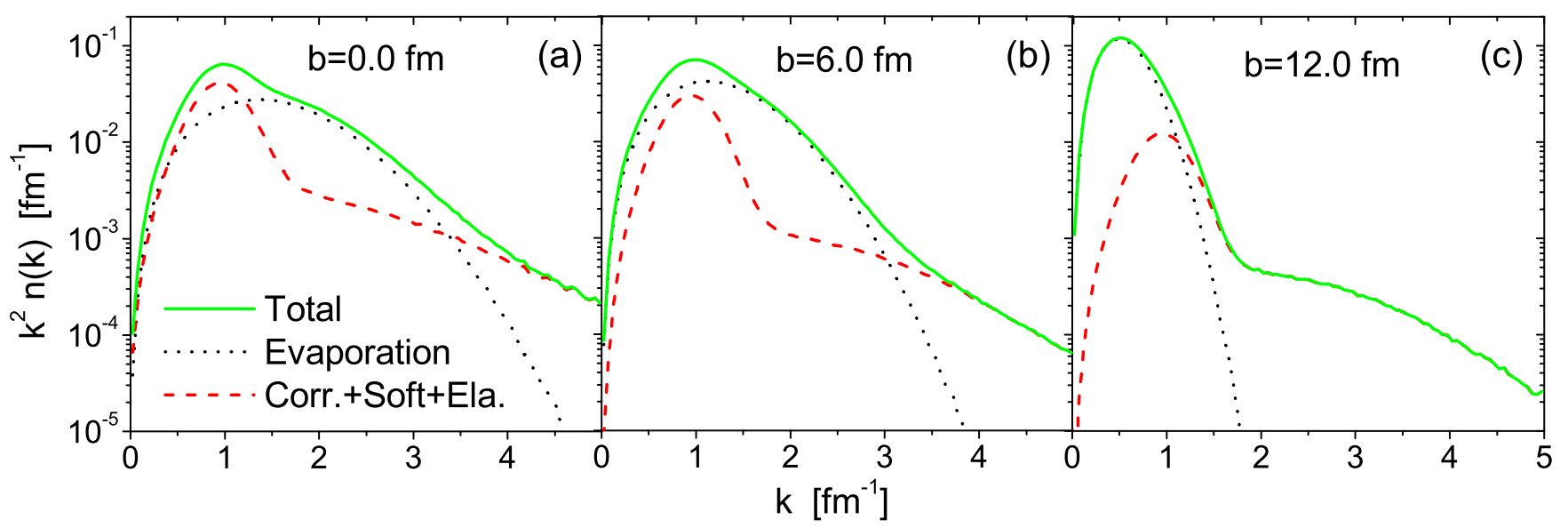

FIG. 8: (Color online) The momentum distributions $k^{2} n(k)$ of emitted nucleons in $P b-P b$ collisions at $P_{L a b}=160$ GeV, calculated within our full model. We show the different contributions due to evaporation (black dotted) and the sum of all other nucleon emission mechanisms, namely soft nucleons from the surface, correlated nucleons and elastically scattered nucleons (red dashed); the total momentum distribution is also shown (green solid). [(a)-(c)] The momentum distributions plotted for impact parameters of $b=0, b=6$, and $b=12 \mathrm{fm}$, respectively. Note that for central collisions the most relevant contribution to the total normalization is due to the high-momentum part, as expected, while when $b$ increases the dominant contribution is due to evaporation.

\section{NUCLEON EMISSION AND MOMENTUM DISTRIBUTIONS}

We have developed a model for nucleon emission as a function of the solid angle $d \Omega=d(\cos \theta) d \phi$ from one of the colliding nuclei in a $\mathrm{Pb}-\mathrm{Pb}$ collision. The model takes into account several different mechanisms for the nucleon emission reflecting the geometry of the high energy $A A$ interaction. Previously only evaporation of nucleons from the excited spectator system resulting from the collision was considered and the total energy released in the emission was treated as an input parameter. In the previous section we developed a novel approach which allows us to calculate the total excitation energy on an event-byevent basis. Part of this energy is emitted in a surface process resulting from the sudden removal of a part of the nucleus with large energies transferred locally to nucleons which had strong bonds with removed nucleons. We first consider emission of the high momentum (Sec. IVA) and low momentum nucleons (Sec. IVB) from the surface. We also consider nucleons emitted in elastic interactions of two nucleons of the colliding nuclei which gives an important contribution for certain emission angles (Sec. IV C). The nucleons generated in the surface emission and elastic scattering propagate with a large fraction of the events through the spectator system and transfer energy to the bulk of the spectator system (Sec. IVD). Taking into account all these effects we evaluate the total energy available for evaporation of soft nucleons (Sec. IVE).

We discuss in detail the different mechanisms and how we calculate the momentum distributions of emitted nucleons in the following subsections.

\section{A. Correlated nucleons emission}

For a given impact parameter $b$, we calculate the interaction between individual nucleons within the Glauber multiple scattering model as described in Sec. [1]and classify the different pairs of the nucleons as described in Sec. III. Next, we consider the mixed pairs, i.e. those pairs in which one of the nucleons is a spectator and the second is an interacting one, and define nucleons as active on the basis of the potential energy transferred to them. These nucleons should be the candidates among which we choose actually correlated nucleons. We have combined information about the short-range character of correlations and the relatively large contribution of correlations to the total potential and kinetic energies. To this end, we define as active the spectators which are, in a given event, at a distance below some $r_{\max }$ from at least one interacting nucleon. We estimate $r_{\max }$ from the following considerations. The SRC originate from the internucleon distances $\leq 1.4 \mathrm{fm}$, hence $r_{\max }>1.4 \mathrm{fm}$. At the same time for $r>2 \mathrm{fm}$ the internucleon potential corresponds to energies significantly smaller than the Fermi energy. Hence a removal of such a long range bond is likely result in collective excitations of the residual system. Hence a reasonable value of this parameter of the model is $2 \mathrm{fm} \leq r_{\max } \leq 1.4 \mathrm{fm}$. In the following we will use as a base value $r_{\max } 2 \mathrm{fm}$. The sensitivity to the 

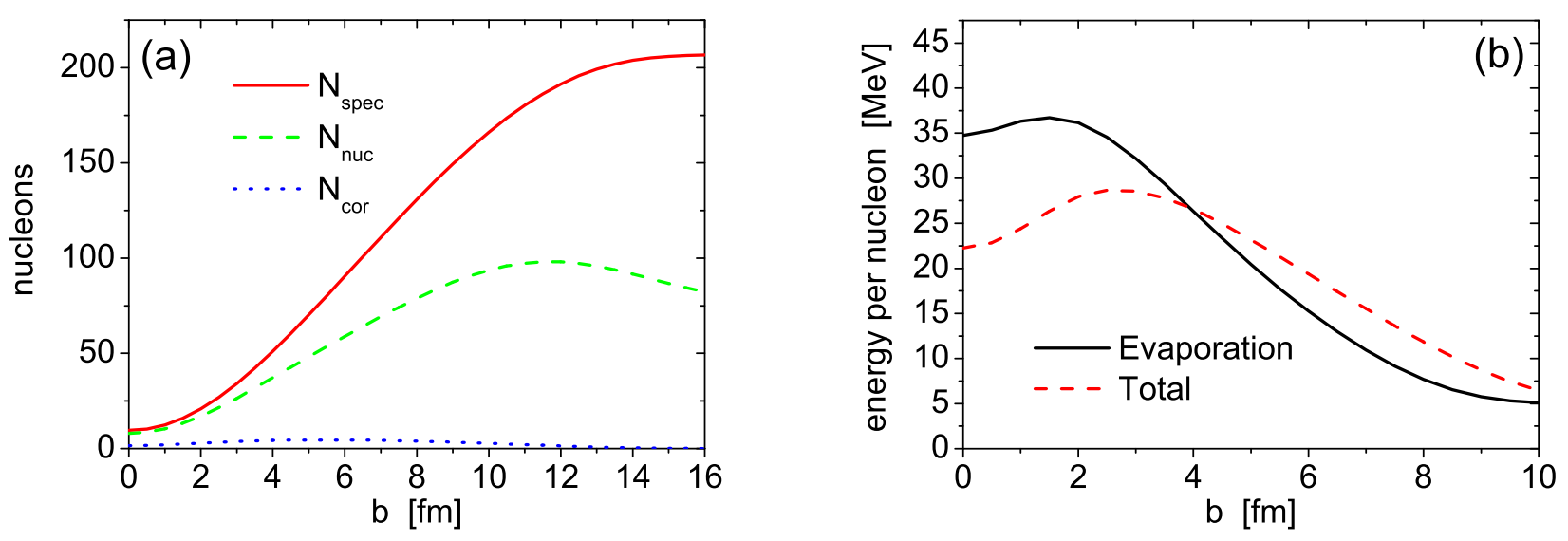

FIG. 9: (Color online) (a) Spectators in a $P b-P b$ collision within a Glauber model at NA49 energy $\left(P_{L a b}=160\right.$ GeV per nucleon). The total number of spectators $N_{\text {spec }}$, unbound nucleons $N_{\text {nuc }}$, and high-momentum nucleons $N_{\text {cor }}$ are shown. Data from the NA49 experiment [1] was used to determine the spectators/emitted nucleons ratio. (b) The kinetic energy per emitted soft nucleon as calculated within our model. We show the case in which only evaporation is taken into account (black solid line) and the case when all the effects described in Sec. IV] are taken into account (red dashed line).

variation of $r_{\max }$ is explored in Sec. IVG Next we calculate the energy transferred to each of the active nucleons, making use of our realistic estimate of the potential energy due to the removal of the interacting nucleons. After ranking the active nucleons starting from the one to which the highest energy is transferred, we choose as correlated the first $25 \%$ of them, according to the estimated fraction of correlations in heavy nucleons [21]. We choose, among the active ones, those nucleons which have larger potential energies and at the same time are at smaller distances from one of their interacting partners, since spatial proximity is a basic requirement for two nucleons to be correlated. This is also clear from the short-range character of correlation functions used in many-body calculations. A delicate feature we need to deal with is that in quantum mechanics there is no one-to-one relation between the potential $V$ and kinetic energy; the relation $T=-V+\epsilon$, with $\epsilon$ the binding energy per nucleon, is valid only in average. Hence we will use a probabilistic algorithm of assigning correlated nucleons random momentum vectors with modulus given by the probability distribution $n_{1}(k)$, which is the correlated, high momentum tail of the momentum distribution from Ref. [21]. In the model of Ref. 21] the total momentum distribution in a nucleus is modeled as a mean field, low momentum part $n_{0}(k)$ plus a correlated, high-momentum part $n_{1}(k)$, which accounts for the $75 \%$ and $25 \%$, respectively, of the total normalization in a heavy nucleus. The procedure will be discussed again in Sec. IVG, after several intermediate steps are described. Note that the production of nucleons from the correlations has forward-backward asymmetry which for moderate momenta is given by the flux factor $\left(1+k_{3} / m_{N}\right)$, where $k_{3}$ is the longitudinal component of the nucleon momentum in its rest frame. So more nucleons are emitted forward (along the beam direction of the projectile) in the rest frame of a nucleus. In this paper to simplify the discussion we consider quantities symmetrized over $k_{3} \rightarrow-k_{3}$.

At the next step active nucleons propagate through the residual system and could be absorbed. This effect is evaluated in Sec. IVD using fits to the $N N$ elastic scattering data [23].

\section{B. Direct emission of uncorrelated nucleons from the inner surface}

The number of correlated nucleons determined in Sec. IVA is only a fraction of active nucleons whose "broken links" with the removed nucleons produce a large fraction of the available energy and which are located at $r \leq r_{\max }$ from at least one of the removed nucleons. The rest of the nucleons, located near the inner surface resulting from the removal of the interacting nucleons, must be considered as escaping the system as well, but with a momentum distribution given by the low-momentum, mean-field part $n_{0}(k)$ of the model of Ref. [21]. This assumption is reasonable since we assume the active nucleons to be emitted with the same momenta they had in the nucleus, but it is clearly an approximation.

\section{Emissions from elastic scattering}

In addition to the spectators which underwent inelastic scattering, there are (primary) nucleons which scattered elastically. We model the probability of high-energy $N N$ elastic scattering $P_{e l}(b)$ in the following way. It must 

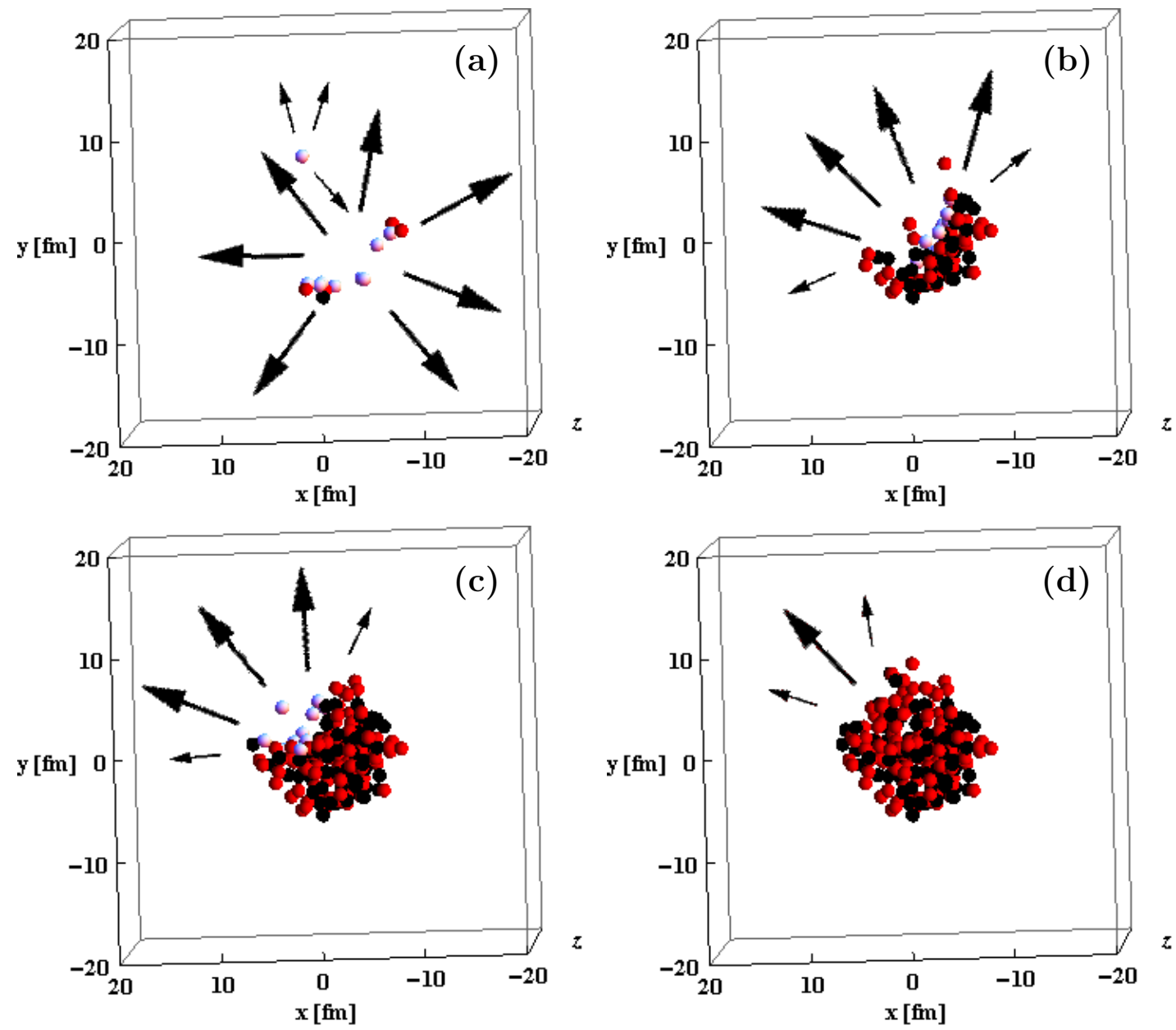

FIG. 10: (Color online) Sketch of the asymmetry of emission of high-momentum, correlated nucleons defined in Eq. (21) and quantitatively evaluated in Fig. 12 in $\mathrm{Pb}-\mathrm{Pb}$ collisions; the preferred direction of emission is shown with arrows as a function of the impact parameter which is oriented as in Fig. 1 and its values through the different panels are $(a) b=1 \mathrm{fm},(b) b=5 \mathrm{fm}$, (c) $b=10 \mathrm{fm}$ and (d) $b=15 \mathrm{fm}$.

obey the sum rule

$$
\int d \boldsymbol{b} P_{e l}(b)=\sigma_{N N}^{e l}
$$

(at NA49 energy we have $\sigma_{N N}^{e l}=5.72 \mathrm{mb}$ ), it must be a function of $b=b_{i j}=\left|\boldsymbol{b}_{i}-\boldsymbol{b}_{j}\right|$ and vanish when $P_{i n}(b)$ does. A reasonable distribution appears to be

$$
P_{e l}(b)=0.077 e^{-5.7(b-1.59)^{2}}
$$

which is compared with $P_{\text {in }}(b)$ of Eq. (3) in Fig. 6. Using Eq. (12), we select elastically scattering nucleons and eventually take into account their recoiling partners as emerging from the event and propagate them through the spectator medium. We calculate the momentum and emission angle of the recoiling nucleon as follows. The target nucleon has momentum $k=\sqrt{k_{t}^{2}+k_{3}^{2}}$ (with probability distribution $n_{1}(k)$ from Ref. [21]); the scattered nucleon has momentum $p=\sqrt{p_{t}^{2}+p_{3}^{2}}$; the fourmomentum transfer $q$ can be assumed to have $q_{0}=q_{3}$ since we are at high energies so $t=q^{2}=q_{0}^{2}-q_{t}^{2}-q_{0}^{2}=$ $-q_{t}^{2} ; t$ can be randomly generated using the elastic scattering amplitude

$$
\frac{d \sigma_{N N}^{e l}}{d t}=\frac{\sigma_{N N}^{t o t}{ }^{2}}{16 \pi} e^{B t},
$$

using the differential $N N$ elastic cross-section data for the energy corresponding to the energy of the $A A$ collisions. This determines $q_{t}$ and $p_{t}=q_{t}+k_{t}$. Since the light-cone fraction, $\alpha$, carried by the nucleon is conserved in the elastic scattering we find

$$
\alpha=1+\frac{k_{3}}{m}=\frac{\sqrt{p_{t}^{2}+p_{3}^{2}+m^{2}}-p_{3}}{m}
$$

leading to

$$
p_{3}=\frac{p_{t}^{2}+m^{2}-m^{2} \alpha^{2}}{2 m \alpha}
$$

This allows to determine the scattering angle $\theta$ is $\tan \theta=$ $p_{t} / p_{3}$. Once elastically scattered nucleon is generated 

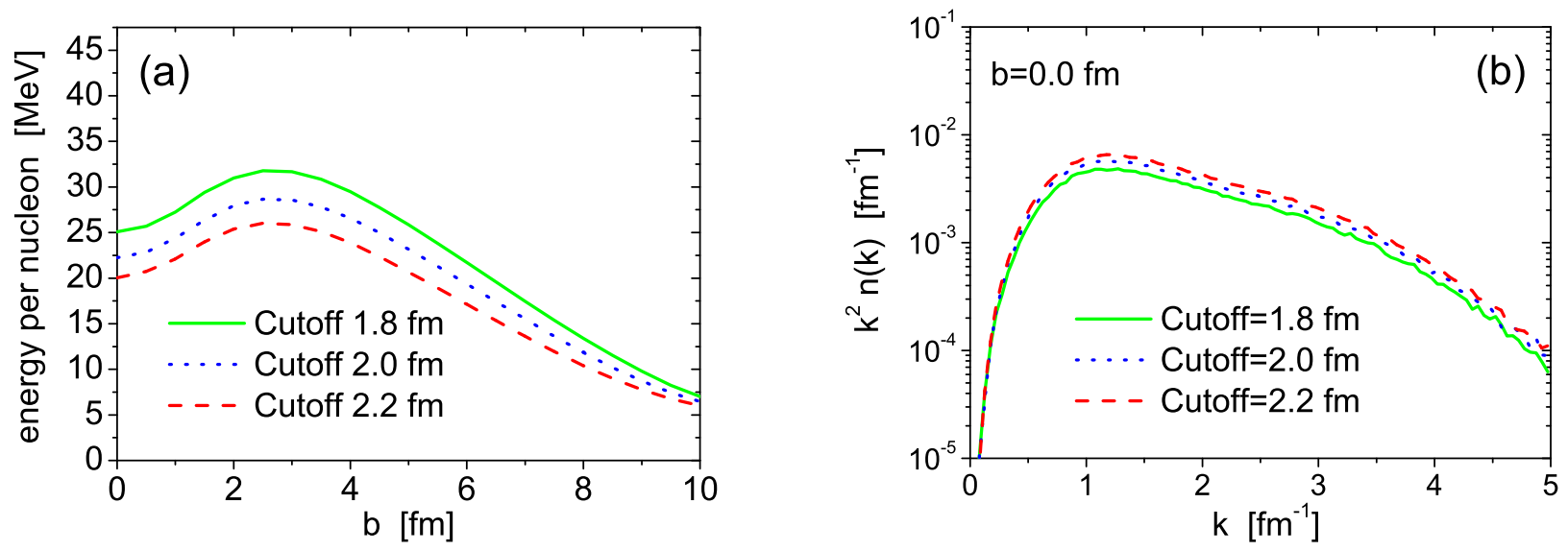

FIG. 11: (Color online) Dependence of (a) the total potential energy per evaporating nucleon and (b) of the correlated contribution to the momentum distribution (b). We show three values of the cutoff distance below which we select active nucleons among the spectator ones. The distance is calculated from the nearest interacting nucleon.

we can use the method described in IVD to determine whether the generated nucleon is absorbed during the propagation, with its energy going into additional heating of the spectator system, or it is emitted with its recoiling momentum, to be collected in the final momentum distribution. Note that the elastic mechanism predominantly contributes for $\alpha \sim 1$. The data from Ref. [12] do indicate an extra contribution at the corresponding kinematics.

\section{Elastic scattering in the final state}

The nucleons which are emitted in the processes described in the previous subsections may be absorbed while propagating through the spectators by thermalization due to elastic rescattering, leading to the heating of the spectator system. We can evaluate the attenuation due to the elastic scattering using $d \sigma_{N N}^{e l} / d T$ of Ref. [23], given as a function of incident nucleon kinetic energy $T=T_{l a b}$ and center-of-mass scattering angle $\theta_{C M}$. We will consider as thermalized those nucleons which, as a consequence of elastic rescattering, are left with kinetic energy smaller than $T_{\min }=\sqrt{k_{\min }^{2}+m^{2}}-m$ kinetic energy, where $k_{\text {min }}=250 \mathrm{MeV} / c$, the typical minimal momentum scale of the SRCs in nuclei. First, we consider as interacting elastically two nucleons whose transverse separation (with respect to the direction of propagation of the nucleon under investigation) is $b_{i j}<\sqrt{\sigma_{N N}^{e l} / \pi}$ with $\sigma_{N N}^{e l}$ evaluated at the corresponding incident momentum, taken from the tabulated values of Ref. 23]. If $k=k_{l a b}=\sqrt{(T-m)^{2}-m^{2}}$ is the emitted nucleon momentum in the nucleus rest frame hitting a spectator nucleon at rest which recoils with kinetic energy $T_{R}$ we have

$$
t=-2 m T_{R}=-2 p^{2}\left(1-\cos \theta_{C M}\right)
$$

where $p=k_{C M}=\sqrt{\frac{1}{2} m \sqrt{k^{2}+m^{2}}-\frac{1}{2} m^{2}}$ is the incident nucleon momentum in the center of mass. Then, the probability for the nucleon emerging from the collision of having more than $T_{\min }=T_{R}-T$ is given by

$$
\int_{40}^{T} d T^{\prime} \frac{d \sigma_{N N}^{e l}\left(T^{\prime}, \theta_{C M}\right)}{d T^{\prime}} / \int_{40}^{\infty} d T^{\prime} \frac{d \sigma_{N N}^{e l}\left(T^{\prime}, \theta_{C M}\right)}{d T^{\prime}}
$$

where the lower limit of integration is dictated by the fact that we have from Ref. [23] tables for $40 \mathrm{MeV}<T<$ $600 \mathrm{MeV}$ and the upper limit can safely be considered large enough. Nucleons with $T<40 \mathrm{MeV}$ are considered as being absorbed the the spectator medium. In Eq. (17) we use $d \sigma_{p p}^{e l}$ both for $p p$ and $n n$ scatterings, and $d \sigma_{p n}^{e l}$ for $p n$ and $n p$. In the case of absorption by the spectator system, we add their kinetic energy to the amount of energy available for the subsequent evaporation. The same procedure has been used to calculate the propagation of the nucleons generated in the primary (high energy) elastic NN scattering (Sec. IVC).

It is worth noting here that to the best of our knowledge the contribution of the high energy elastic scattering mechanism with or without subsequent absorption was not considered before. Of separate interest here is that we calculate this effect (like all other quantities defined in this work) as a function of the $A A$ impact parameter $b$. 

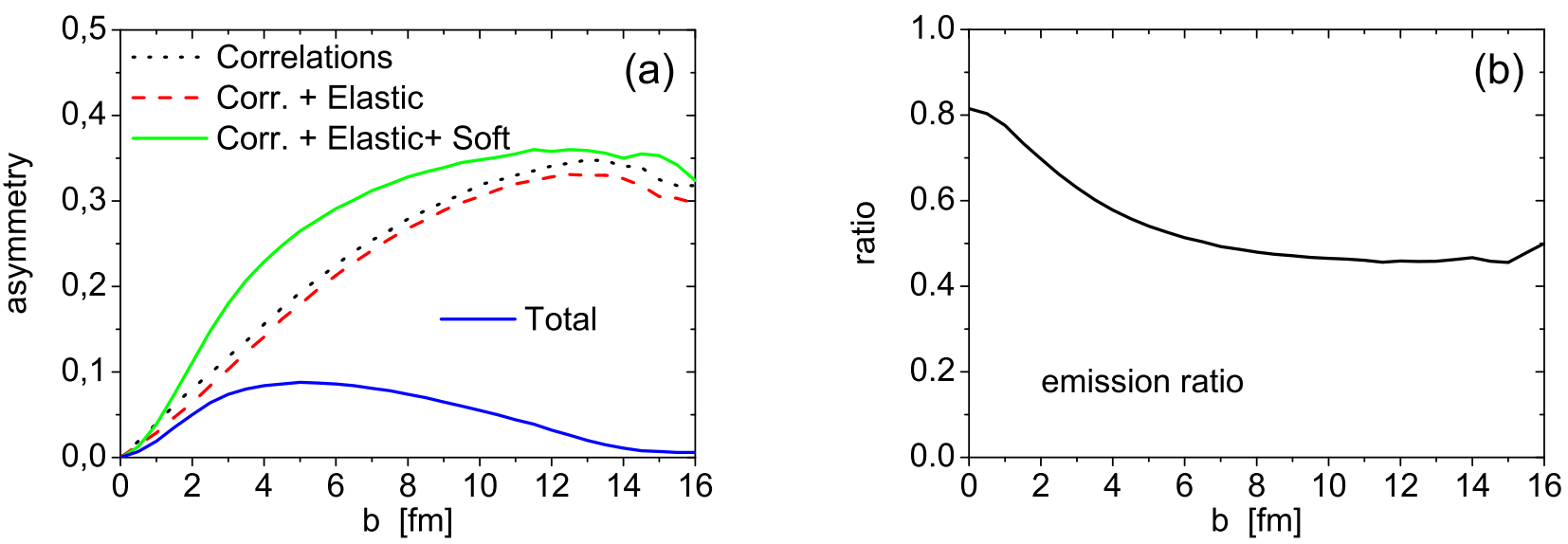

FIG. 12: (Color online) (a) The asymmetry defined in Eq. 21) within the approximations described in Sec. IV the curve labeled with Total correspond to the inclusion of both correlated and soft nucleons from the surface, as well as elastic scattering, absorption and soft nucleons from evaporation. Correlations, soft nucleons from the surface and elastic scatterings exhibit a strong asymmetry, while soft nucleons from evaporation are emitted isotropically. (b) The fraction of emitted high-momentum nucleons which survive after scattering off the spectator system, disregarding any angular dependence.

\section{E. Evaporation mechanism}

A standard mechanism for description of the spectator emission in the process of fragmentation is the excitation of the residual system and subsequent evaporation of nucleons with the exponential probability for the emitted nucleon kinetic energy $T_{n}=p_{n}^{2} / 2 m$

$$
P\left(T_{n}\right)=e^{-T_{n} / T_{0}},
$$

where $T_{0}$ is typically assumed to be close to the average kinetic energy for the Fermi gas [24]. We can estimate the kinetic energy $T_{0}$ for a given value of the impact parameter in the following way. We have calculated the energy released by destruction of nucleon-nucleon pairs in Sec. III] This energy must be distributed among the evaporating nucleons according to the following energy balance equation:

$$
N_{\text {nucl }}\left(T_{0}+\epsilon\right)=-V^{\prime}-T_{\text {emit }},
$$

where $N_{\text {nucl }}$ is the number of evaporated soft nucleons and $T_{\text {emit }}$ is the kinetic energy of the correlated and uncorrelated nucleons emitted from the surface which we discuss in Secs. IVA and IVB The renormalization of $V$ in the right hand side $V^{\prime}=V\left(1+\epsilon /<V>_{N}\right)<0$ reflects the energy released, as described in Sec. III corrected for the binding effects of the removed nucleons. Equation (19) states that the amount of energy $\epsilon$ must be spent for each of the emitted nucleons, and the available energy is provided by the calculated potential energy; note that $N_{\text {nucl }}$, the number of emitted nucleons, and $V$ depend on the $A A$ impact parameter $b$, as does the energy per emitted soft nucleon, $T_{0}$.
The energy balance expressed by Eq. (19) must be corrected for several additional effects. First, we have to take into account that a significant fraction of the nucleon spectators produced in the decay of the spectator system are bound in light $A=2 \div 4$ and heavier nuclear fragments. As a result one has to replace in Eq. (19) $N_{\text {nucl }}$ by $N_{\text {eff }}=N_{\text {nucl }}+K N_{\text {frag }}$ where $N_{\text {frag }}$ is the total number of nucleons bound in the $A \geq 2$ fragments. To determine this factor we use the measurements of Refs. [1] and [12]. We extracted from the data what fraction of the nucleons belongs to the fragments and found it to be $\approx 30 \%$, leading to $K \approx 0.3$. Hence we did not include it explicitly in the energy balance of Eq. (19). (The experiments were performed using light projectiles, and neutron production was not measured. We made a natural assumption that for collision of light nuclei the proton and neutron spectra coincide. We also assumed that the fraction of energy in the fragments remains approximately the same for collisions of heavy nuclei.)

There are other effects to be accounted for in Eq. (19) leading to a modification of the initial transferred energy to be eventually available for evaporation. The final excitation energy is obtained starting with the initial transferred energy calculated by considering the broken potential bonds due the removal of nucleons, as described in Sec. III. In Eq. (20) we kept track of contributions which decrease the available energy. Namely we took into account that if a correlated or uncorrelated nucleon is emitted from the region close to the interaction surface, its kinetic energy is subtracted from the available energy; on the other hand, if the same nucleon is absorbed by the spectator system while propagating through it, the corresponding energy is put back into the total. We included also the contribution to the evaporation energy of the nu- 
cleons produced in the primary elastic scatterings which were absorbed by the spectator system. The energy per evaporating nucleon $T_{0}$ is then obtained by replacing Eq. (19) with

$$
N_{e f f}\left(T_{0}+\epsilon\right)=-V^{\prime}-T_{e m i t}+T_{a b s},
$$

with $T_{\text {emit }}$ and $T_{a b s}$ the total energies due to nucleon emission and absorption in the final state, respectively.

The same procedure is used to determine the momentum distributions of produced nucleons. When nucleons are emitted from the interaction surface, they are assigned a momentum $k$ with probability distribution $n_{0}(k)$ or $n_{1}(k)$ if they are uncorrelated or correlated, respectively. If they survive propagation through the spectators their momentum is collected. The same algorithm is used for the nucleons experiencing the elastic scattering in the initial state. These nucleons are assigned initial momenta with probability $n_{0}(k)+n_{1}(k)$. Momentum distribution and other results will be presented in the following.

\section{F. Results of calculations}

Results for the calculation of the momentum distributions are shown in Figs. 7 and 8 . The curves correspond to the inclusion of the different effects we have described in this section. The curve labeled Evaporation corresponds to the energy per emitted nucleon obtained by dividing the total potential energy by the number of the expected free nucleons. The curve labeled with Total is obtained from Eq. (20), where we account for (i) the energy going into the kinetic energy of the emitted particles $T_{\text {emit }}$ and the kinetic energy of the absorbed particles $T_{a b s}$ going into additional available energy and (ii) the number of nucleons emitted in the first stage from the surface close the interaction. These are either highmomentum or soft nucleons. The number of nucleons emitted from the surface is subtracted from the number of free nucleons which are produced in the evaporation. The curves labeled Correlation correspond to the inclusion of high-momentum-correlated nucleons; curves Soft correspond to the inclusion of soft nucleons emitted from the surface, which are the nucleons left after subtracting the correlated from the active ones; the curves labeled Elastic correspond to the contributions from primary elastically scattered nucleons. Note that subsequent absorption from the residual system by elastic rescattering is taken into account for all the separate contributions. The results of our calculation of the energy per evaporating nucleon are shown in Fig. 9 as a function of the impact parameter 9(b)]. Both the result including only evaporation and total result [Eq. (20)] are given. Figure 9(a) shows the dependence of the number of interacting and spectator nucleons on $b$ of the number of spectators.

We would like to comment on data from Ref. [12]. Momentum distributions of detected nucleons were explicitly measured; nonetheless, a direct comparison with our results may not be trivial. The authors have compared the observed momentum distribution with the theoretical momentum distribution in nuclei, which is not consistent with the observation that (i) most of the nucleons are emitted by the evaporation mechanism, whose distribution is given by Eq. (18) and it is expected to differ significantly from the momentum distribution of nucleons inside the nucleus and (ii) the observed momentum distribution depends on the transferred energy in the process of removing one nucleon while the momentum distribution is the integral of the spectral function for one nucleon removal over all the removal energy range; a calculation of the effects of the integration of the spectral function on a limited region of energy may be found in Ref. [25]. (iii) We suggest that only the high-momentum part of the observed momentum distribution can be compared with the nucleon momentum distribution in nuclei $n(k)$, since it is due to direct emission of correlated nucleons whose momentum may be distorted by the propagation through the spectator system but still will be similar to the original $n(k)$. Moreover, the high-momentum region of the observed momentum distribution is also affected by primary elastically scattered nucleons, which must be taken into account. Data from Ref. [12] exhibits a change in slope of the momentum distribution of protons as well as a strong change of the shape of the transverse-momentum distribution for nucleons with total energy equal to $E_{A} / A$. The first effect may be due to the high-momentum, correlated nucleons, as suggested by results of calculations shown in Fig. 7 (b). While the second effect is likely to be due to the elastic-scattering mechanism.

\section{G. Sensitivity to the parameter $r_{\max }$}

Here we return to discussion of $r_{\max }$ and sensitivity of the results to a specific choice of $r_{\max }$ which played an important role in identifying the active nucleons. We already gave our rationale for the choice of $r_{\max } \approx 2 \mathrm{fm}$. An alternative way to determine $r_{\max }$ is to require that it is equal to the average maximum distance from the nearest interacting nucleons of spectators with potential energy larger than the average $\langle V\rangle / A$. This definition leads to the value of $r_{\max }=2 \mathrm{fm}$, which is close to our qualitative expectations. To explore the sensitivity to this parameter we varied it between 1.8 and $2.2 \mathrm{fm}$. The results are shown in Fig. 11. It can be seen that the final result for the energy per evaporating nucleon [11(a)] changes by at most $10 \%$, with an even smaller effect on the momentum distribution for $k<300 \mathrm{MeV} / c$ 11(b)]. The effect is larger for high momentum nucleons. However, it should be stressed that reducing $r_{\max }$ to less than $2 \mathrm{fm}$ would produce an unrealistically low number of correlations, since the $N N$ correlation functions of Eq. (17) have been shown by many-body calculations (see the correlation functions in Refs. [17] and [27] and references therein) to extend up to relative distances of at least 2 fm. Also, we checked that the variation of $r_{\max }$ leads to 

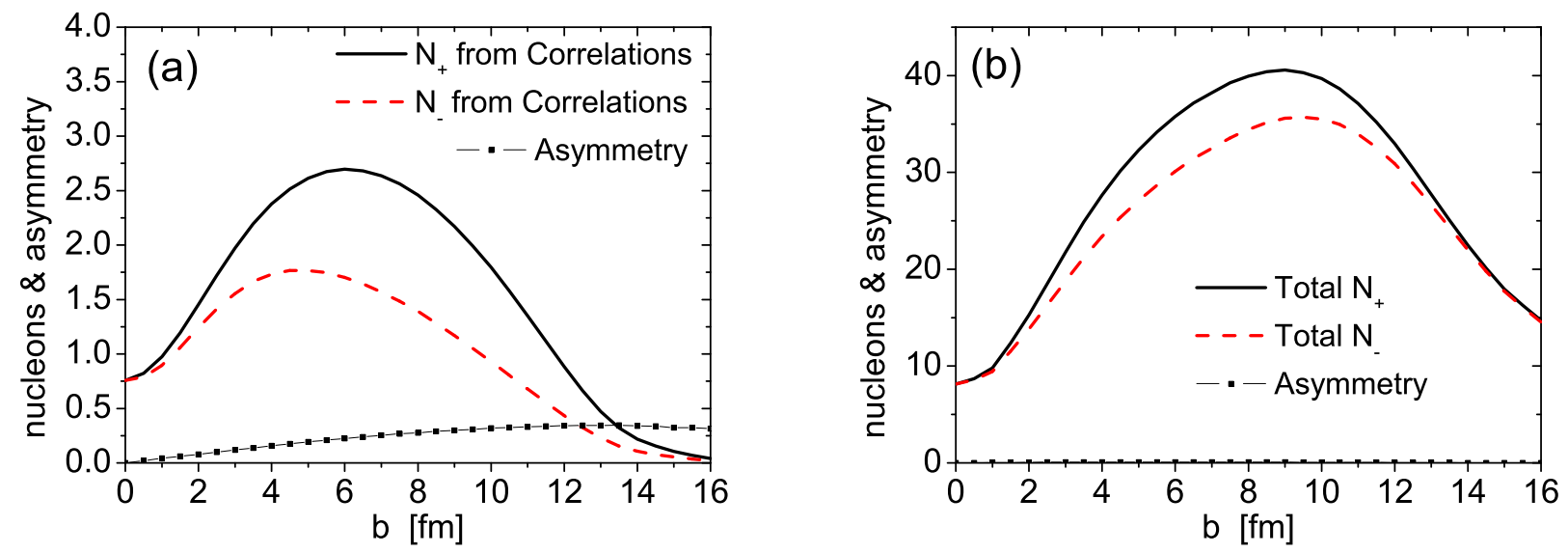

FIG. 13: (Color online) The number of high-momentum nucleons emitted in the hemisphere containing the projectile $\left(N_{+}\right.$; solid curve) and in the opposite one ( $N_{-}$; dashed curve); line and symbol curve represent the asymmetry defined in Eq. (21). (a) Only correlated nucleons; (b) quantities calculated using our model with all the different mechanisms of nucleon emissions, including soft nucleons from evaporation, as described in Sec. [V]

a very small modification of the asymmetry of emission, which we discuss in the next section.

\section{ANGULAR DEPENDENCE OF DIRECTED FLOW OF EMITTED HIGH-MOMENTUM NUCLEONS}

One of the aims of this work is to estimate the asymmetry of emerging from the $A A$ collision. These nucleons are generated on the inner surface left after the fast propagation of the projectile nucleus; we define $N_{+}$to be the number of nucleons ending up in the hemisphere oriented toward free space and $N_{-}$its analog in the opposite hemisphere. We can define the impact parameter-dependent asymmetry as follows:

$$
A(b)=\frac{N_{+}-N_{-}}{N_{+}+N_{-}} .
$$

We expect that the few nucleons surviving in a central collision will find little or no matter to propagate through in any direction, resulting in $A(0)=0$. The situation is depicted in Fig. 10, for different impact parameters ranging from $b=1 \mathrm{fm}$ (top left panel) to $b=15 \mathrm{fm}$ (bottom right panel). In the first case of almost central collisions, few nucleons are left; they are far from each other and free to propagate. For increasing $b$, it can be seen how an asymmetry arises in the rest frame of the target nucleus, since nucleons are produced with random momentum direction and they can end up propagating into free space or through the spectator system which prevents emitted nucleons to propagate freely. For a large impact parameter, the asymmetry of Eq. (21) which is due only to the correlations should approach the value given by taking $N_{-} \simeq 0.5 N_{+}$, which is the fraction of nucleons surviving after the scatterings through the spectator matter at large impact parameters. This would result in $A(b) \simeq 0.3$. The calculations of $A(b)$ performed within our model are shown in Figs. 12 and 13 and confirm these expectations. Figure 12 shows the asymmetry including nucleons from the different emission mechanisms. The contribution due to correlations is, as already stated, strongly asymmetric; elastically scattered nucleons lead to similar asymmetry as they also originate from the surface; soft nucleons originating from the surface somewhat increase asymmetry. The largest contribution, as far as the number of nucleons is concerned, is from the soft, evaporating ones, and makes the total asymmetry much smaller. Figure 13 shows the individual contributions to $N_{+}$and $N_{-}$from correlated nucleons alone and from the total number of emission of spectator nucleons. Note that for $b>12 \mathrm{fm}$ we used an extrapolation of the NA49 data [11] for the number of nucleons produced at a given impact parameter for determining the fraction of energy taken from nucleons and fragments, as described in Sec. IV.

In this analysis we did not take into account the effect of the Coulomb interaction between nuclei which gives fragmenting systems of opposite transverse momenta. The effect gives an asymmetry of the opposite sign than the effect we discussed here. Our effect is large for large nucleon momenta, while the Coulomb effect is most important for the lowest momentum neutrons. We will consider the Coulomb effect elsewhere.

We mentioned in the Introduction that RHIC experiments observed displacement of the core of the neutron shower from the center of the detector. However, this effect is dominated by low-momentum neutrons and may be more sensitive to the Coulomb effect. Hence it would 
be important to study asymmetry separately for different ranges of the neutron momenta as well as study experimentally correlation of the strengths and directions of asymmetries of two fragmentation regions. When the origin of the asymmetry is cleared out it would be possible to use this effect to solve the ambiguity in the sign of the impact parameter $\boldsymbol{b}$. Note here, that the correlation of $v_{1}$ with corresponding asymmetry for hadrons at the rapidities away from the central one was observed at RHIC. However due to the lack of understanding of the origin of the neutron asymmetry it was impossible to use this observation to constrain the current models of $A A$ collisions at RHIC.

We investigated the dependence of the $A(b)$ on the cutoff distance from the closest interacting nucleon, as discussed in the previous section. We find negligible effects on the asymmetry. This is due to the fact that the emission of correlated nucleons is highly asymmetric, but the asymmetry is dominated by isotropic emissions in any case so the small relative fraction of correlations practically does not affect $A(b)$.

\section{COMPARISON WITH THE ABRASION-ABLATION MODEL}

In this section we compare our novel approach for the calculation of the excitation energy of the spectator system with the one employed in Ref. [1]. In the cited Ref. [1], the authors use the abrasion-ablation model for hadronic interactions and the relativistic electromagnetic dissociation model for electromagnetic interactions of relativistic heavy ions to describe data of charge-changing cross section in $\mathrm{Pb}-A$ collisions. In particular they address the question of how to estimate the excitation energy of a nuclear system formed by sudden removal of several nucleons, which is also one of the main aims of the present work, and describe the decay of excited nuclear systems within the statistical multi-fragmentation model. The abrasion model describes participant and spectator nucleons with participants originating from the overlapping parts of the colliding nuclei, while their nonoverlapping parts are treated as spectators which represent excited remnants of the initial nuclei which undergo secondary decay by statistical evaporation and fission models in the so-called ablation model.

The $N N$ interaction probability in Ref. [1] is defined through (uncorrelated) nuclear thickness functions, while in our approach correlations are taken into account automatically by using improved configurations from Ref. 7]. The main difference then consists in the estimate of the spectator system (prefragment, in the terminology of Ref. 1]) excitation energy. We use similar values of $N N$ cross sections. The authors of Ref. [1] describe excitation energy by the abrasion model, which basically evaluate the energy due to a hole in the uncorrelated ground state of the initial nucleus, while we use the realistic calculation of Sec. III moreover, in Ref. [1] it is explicitly mentioned that the procedure is not well defined for a large number of removed nucleons and that their method of calculating excitation energies of prefragments via the hole state densities should be considered only as a model assumption. A comparison with other approaches was also done in Ref. [1], finding for the excitation energy per nucleon a value of $\simeq 40 \mathrm{MeV}$ using the ablation model and $\simeq 27 \mathrm{MeV}$ using a more refined formula, the latter estimate being confirmed by data; we note that the value of $\simeq 27 \mathrm{MeV}$ is in good agreement with our findings of Fig. 9 where this value is just about our estimate for $b \simeq 3-4 \mathrm{fm}$; we believe that one of the important improvement of our approach is precisely the possibility of giving an impact-parameter dependent estimate of the excitation energy, which was untouched by any of the previous approaches. As a last remark, let us stress that in Ref. [1] it was mentioned that their model should be complemented with FSI, which we take into account in our estimate of the energy per nucleon, and the additional energy brought in by elastically scattered primary nucleons subsequently absorbed by the spectator system, which we also considered.

\section{CONCLUSIONS}

We have demonstrated that the underlying dynamics of the process of the nucleus fragmentation in high energy nucleus-nucleus collisions is strongly related to the the presence of short-range correlations in nuclei. We predict a number of new phenomena which could be tested in the current and forthcoming heavy ion experiments, including the dependence of the momentum spectrum on the impact parameter strong asymmetry of the emission nucleons along the $b$ direction. Such an asymmetry as well as other predicted effects may be of use for more detailed analyses of dynamics of the heavy ion collisions. In particular, it would allow one to investigate whether similar asymmetry is present for the hadrons produced in $A A$ collisions away from zero center of mass rapidity. We also predict a close connection of the spectrum of nucleons in the central collisions and momentum distribution in the nuclei. We are now in the process of implementing the discussed effects in a complete MC event generator of $A A$ collisions and results will be presented elsewhere [26].

\section{ACKNOWLEDGEMENTS}

We thank G. Bertsch, T. Csorgo, L. Frankfurt, I. N. Mishustin, I. A. Pshenichnov, M. Vargyas, S. Voloshin and S. White, for very useful discussions. This work is supported by a DOE grant under contract number DE-FG02-93ER40771. During substantial revision of the manuscript M.A. was supported by the project HadronPhysics2 of the FP7 of the EU (Grant No. 227431). M.A. thanks the HPC-Europa2 Consor- 
tium (project number: 228398), with the support of the EC - Research Infrastructure Action of the FP7 - and
EPCC, Edinburgh for the use of computing facilities.
[1] C. Scheidenberger et al., Phys. Rev. C 70, 014902 (2004).

[2] R. Subedi, R. Shneor, P. Monaghan, B. D. Anderson, K. Aniol, J. Annand, J. Arrington, H. Benaoum et al., Science 320, 1476-1478 (2008).

[3] R. Shneor et al. [ Jefferson Lab Hall A Collaboration ], Phys. Rev. Lett. 99, 072501 (2007).

[4] E. Piasetzky, M. Sargsian, L. Frankfurt, M. Strikman, J. W. Watson, Phys. Rev. Lett. 97, 162504 (2006).

[5] A. Tang, J. W. Watson, J. L. S. Aclander, J. Alster, G. Asryan, Y. Averichev, D. Barton, V. Baturin et al., Phys. Rev. Lett. 90, 042301 (2003).

[6] L. Frankfurt, M. Sargsian and M. Strikman, Int. J. Mod. Phys. A 23, 2991 (2008).

[7] M. Alvioli, H. -J. Drescher, M. Strikman, Phys. Lett. B680, 225-230 (2009).

[8] W. Broniowski, M. Chojnacki and L. Obara, Phys. Rev. C 80 (2009) 051902

[9] W. Broniowski and M. Rybczynski, Phys. Rev. C 81, 064909 (2010)

[10] http://www.phys.psu.edu/ malvioli/eventgenerator

[11] H. Appelshauser et al. [NA49 Collaboration], Eur. Phys. J. A 2, 383 (1998).

[12] L. Anderson et al., Phys. Rev. C 28, 1224 (1983).

[13] J. Aichelin, Phys. Rept. 202, 233 (1991).

[14] S. Afanasiev et al. [PHENIX Collaboration], Phys. Rev. C80, 024909 (2009).
[15] J. Adams et al. [STAR Collaboration], Phys. Rev. C73, 034903 (2006).

[16] B. I. Abelev et al. [STAR Collaboration], Phys. Rev. Lett. 101, 252301 (2008).

[17] M. Alvioli, C. Ciofi degli Atti and H. Morita, Phys. Rev. C 72, 054310 (2005)

[18] X. N. Wang and M. Gyulassy, Phys. Rev. D 44, 3501 (1991).

[19] A. Bialas, M. Bleszynski, W. Czyz, Nucl. Phys. B111, 461 (1976).

[20] M. Alvioli, C. Ciofi degli Atti and H. Morita, Phys. Rev. Lett. 100, 162503 (2008)

[21] C. Ciofi degli Atti and S. Simula, Phys. Rev. C 53, 1689 (1996)

[22] D. S. Koltun, Phys. Rev. C 9, 484 (1974).

[23] R. A. Arndt, W. J. Briscoe, I. I. Strakovsky and R. L. Workman, Phys. Rev. C 76, 025209 (2007) and private communication

[24] H. Feshbach and K. Huang, Phys. Lett. B 47, 300 (1973).

[25] C. Ciofi degli Atti, E. Pace and G. Salmè, Phys. Lett. B 141, 14 (1984)

[26] M. Alvioli, M. Striman, S. White, M. Vargyas, A. Ster and T. Csorgo, in preparation

[27] W. J. W. Geurts, K. Allaart, W. H. Dickhoff et al., Phys. Rev. C54, 1144-1157 (1996). 